\title{
Sociolinguistic context, not test language, predicts implicit bias scores between ethnicities*
}

\author{
Lindy Comstock ${ }^{\mathrm{a}, \dagger}$ and Marco Iacoboni $\mathrm{i}^{\mathrm{b}, \mathrm{c}, \mathrm{d}, \mathrm{e}}$ \\ a Department of Life Sciences Core Education, University of California, Los \\ Angeles, Los Angeles, CA, United States \\ ${ }^{b}$ Ahmanson-Lovelace Brain Mapping Center, David Geffen School of Medicine, \\ University of California, Los Angeles, Los Angeles, CA, United States \\ ${ }^{c}$ Semel Institute for Neuroscience and Human Behavior, David Geffen School of \\ Medicine, University of California, Los Angeles, Los Angeles, CA \\ ${ }^{d}$ Department of Psychiatry and Biobehavioral Sciences, David Geffen School of \\ Medicine, University of California, Los Angeles, Los Angeles, CA \\ ${ }^{e}$ Brain Research Institute, David Geffen School of Medicine, University of \\ California, Los Angeles, Los Angeles, CA, United States
}

\begin{abstract}
Language plays a complex role in mediating implicit associations. This study shows that the degree to which these associations are a state-dependent or stable construct may be the product of life experiences. Mexican-American bilinguals were cued regarding the social acceptability of mixing languages ('codeswitching'). A subset of them-according to a latent profile analysis revealing five response patterns that significantly correlated with participant background characteristics-shifted or even reversed their implicit attitudes toward mixing languages. Thus, language does not "predetermine" bias, as previously claimed, but may evoke contradictory associations within the same individual, indexing shifting context-dependent relationships. The varied response to the same linguistic manipulation suggests that implicit attitudes reflect deeper conceptual processing rather than shallow linguistic associations.
\end{abstract}

Keywords: Implicit Associations, Ingroup Devaluation, Intergroup Dynamics, Bilingualism, Stereotyped Attitudes

Statement of Relevance: Findings target the underpinnings of "false consciousness," a phenomenon in which minorities internalize self-depreciating societal values and perpetuate stereotypes against their own ingroup. We investigate ingroup devaluation among Mexican-Americans - a prominent minority group with a widespread presence throughout the United States - with the aim to identify contextual triggers that evoke shifts in implicit bias against minorities and potential underlying factors that may predispose certain subpopulations to engage in or be resilient against ingroup devaluation. Identifying factors associated with implicit bias against ethnic and linguistic minorities is an endeavor of great importance to the public at large, as such biases underlie many of the pressing issues found in the current U.S. social and political landscape. Revealing how attitudes towards language use selectively prime implicit bias can make possible the fight against perpetuating negative implicit associations in public discourse.

\footnotetext{
${ }^{*}$ None of the authors have potential conflicts to disclose.

$\dagger$ This is to indicate the corresponding author.

Email addresses: lbcomstock@ucla.edu (Lindy Comstock), iacoboni@ucla.edu (Marco Iacoboni)
} 


\section{Introduction}

The choice to use a particular language variety reflects the implicit psychological associations a speaker has formed between social settings and social relationships, informed by past experiences with the linguistic communities he or she inhabits (Molinsky, 2007; Myers-Scotton, 1995). In previous attempts to measure the effect of language choice on implicit attitudes, researchers have argued that language mediates the accessibility of social representations, and correspondingly, their expression in the Implicit Association Test (IAT) (Greenwald, Nosek, \& Banaji, 2003). These studies found that the same cohort of bilingual participants shifted their implicit attitudes between linguistically-distinct ethnic groups when assessed on the same task in two languages, leading researchers to conclude that language plays a key role in constructing implicit associations in real time as part of a state-dependent process (Danziger \& Ward, 2010; Ogunnaike, Dunham, \& Banaji, 2010). The present study suggests that language is more than just a selective filter that determines which associations are activated; instead, language indexes more complex, context-dependent sociolinguistic relationships to convey information that is socially relevant, such as an ethnic or institutional affiliation, within a particular contextual frame.

Linguistic codeswitching - the "mixing" of languages within a conversation or sentence - constitutes a social behavior deeply rooted in the unconscious knowledge of context-specific social norms. In the United States, codeswitching has often been described in pejorative terms by the monolingual majority, who may associate the use of languages other than English with a racialized, non-normative outgroup status, particularly within institutional settings (Boztepe, 2005; Drake, 1977; MacSwan, 2000). Yet multilingual communities typically welcome the creative expressivity of codeswitching among family and friends as an expression of ingroup affiliation (De Fina, 2007; Genesee, 1982; Kharkhurin \& Wei, 2015). Because Mexican-American heritage language speakers in the U.S. grow up experiencing a conflict in the sociolinguistic attitudes to language use professed at home and in public institutions, they form expectations about where and with whom codeswitching is appropriate. Therefore, we predicted that Mexican-American bilinguals' implicit attitudes would shift not in response to the language of the experimental task, but in response to the social context in which an ethnically marked language practice, such as codesswitching, occurs within that task. 


\section{Method}

Mexican-American bilinguals in Southern California are immersed in a multicultural environment that renders them cognizant of context-dependent expectations about codeswitching. Participants included 85 (68 female) undergraduate heritage language speakers of Mexican Spanish between the ages of 18-27 $(\mathrm{M}=20.18, \mathrm{SD}=1.36)$ who were born in Los Angeles or moved to the area with their family before the age of five. Participants were required to exhibit fluency and confirm their daily use of Spanish and English. Written informed consent was collected and participants received course credit upon completion of the experiment. Sample size was determined based on IAT metaanalysis data and recommended best practice for latent profile analysis (Masyn, 2013). Even significant small effect sizes are commonly obtained in IAT studies with 41 participants (Oswald, Mitchell, Blanton, Jaccard, \& Tetlock, 2015) and between 75100 participants should allow for a true latent structure to be recovered given our model design features (Park \& Yu, 2018).

\subsection{Surveys and Pretests}

Participants' sociolinguistic background characteristics were assessed through a mixture of commonly implemented self-report surveys and objective measures. Selfreport data was gathered in the Language \& Social Background Questionnaire (Anderson, Mak, Chahi, \& Bialystok, 2018); a questionnaire based on Intergroup Contact Theory (Pettigrew, 1998) in which participants reported a) the ethnicity of their 5 closest friends, b) the frequency of which they mix languages (codeswitch) with their closest friends (always, usually, sometimes, rarely, never), c) how many (none, a few, many, most, or all) European Caucasian or Mexican Latino people comprise their formal and informal social circles (romantic partners, friends, classmates, work colleagues, work supervisors, and teachers), and d) the frequency (always, usually, sometimes, rarely, never) with which they codeswitch in formal and informal contexts (with friends, family, social media, classmates, work colleagues, work supervisors, teachers); and a "feelings thermometer" (Judd, Park, Ryan, Brauer, \& Kraus, 1995) measuring how "warm" participants felt towards a) European Caucasian and Mexican Latino ethnicities, and b) Spanish, English, and codeswitching. Objective measures included a test of formal Spanish knowledge abridged from the UCLA Spanish \& Portuguese Department placement exam for heritage language speakers and participant ratings of their preference for codeswitched and English-only sentences ( 24 each) on a 7-point Likert scale. 


\subsection{Implicit Association Tests}

The IAT measures implicit associations by alternately pairing positive and negative category labels with the labels of two categories of interest: in this case, European Caucasian and Mexican Latino ethnicity. Participants were asked to sort pictures of the two ethnicities and positive and negative trait words under their corresponding label as fast as possible by means of a button press. In each block the response key for either positive or negative traits is shared by one of the ethnicities, such that the association between the trait and the ethnicity can be calculated by comparing the difference in response time latency between blocks. Five identical IAT versions were constructed according to established best practices for the IAT (Greenwald et al., 2003), differing only by the language in which trait words were presented and the presence or absence of a statement representing a sociolinguistic language attitude prior to commencing the test. The order of the five test versions was counterbalanced across participants, although order effects are minimal between the first and second IAT and absent between the second and subsequent tests (Nosek, Greenwald, \& Banaji, 2005). The IAT has been found to yield good test-retest reliability for trait-dependent, but not statedependent associations (Banaji, 2013). All versions contained pictures of the same 12 individuals, matched for age range and level of attractiveness across the two ethnicities. The pictures were independently identified by a Mexican-Latino volunteer as of either European Caucasian (3M/3F) or Mexican Latino (3M/3F) ethnicity.

The English, Spanish, and bilingual IAT versions were not preceded by a language attitude cue. The English and Spanish versions contained the same words in each language for positive traits (good, joy, love, peace, wonderful, pleasure, glorious, laughter, happy, friendly; bondad, alegría, amor, paz, maravilloso, placer, glorioso, risa, contento, amistoso) and negative traits (bad, agony, repulsive, unfriendly, disgusting, evil, atrocious, failure, hurt, dissatisfied; malo, agonía, repulsivo, antipático, asqueroso, malvado, atroz, fracaso, herido, insatisfecho). The bilingual version, which approximates the practice of codeswitching, presented the same trait words in either English or Spanish; their selection was random. Each block consisted of 20 practice trials and 20 test trials.

The aim of the final two IAT versions was to compare the effect of opposite language attitude cues to codeswitching. The term 'prescriptive linguistics' refers to the enforcement of one standard way of speaking, imposed as the only "correct" way to speak. Prescriptive norms are associated with monolingual language use and a pejorative view of codeswitching. 'Descriptive linguistics' upholds the attitude that 
language norms should be permissive, describing real language use such as codeswitching without judgement. The prescriptive and descriptive IAT versions comprised the bilingual version preceded by a statement corresponding to each language attitude: "Good language is keeping languages separate and pure" (prescriptivism), "Good language is mixing languages creatively" (descriptivism).

\section{Results}

\subsection{Implicit Association Tests}

IAT $D$ scores were calculated according to Greenwald et al. (2003) with positive scores indicating an implicit preference for Mexican Latino (ML) ethnicity over European Caucasian (EC) ethnicity. The $D$ score is considered comparable to effect sizes measured with Cohen's d (Greenwald et al., 2003). An analysis of variance between subjects with the factor of IAT test version yielded no significant result, indicating that the population of subjects as a whole showed no common trend in the relation between their $D$ scores and different IAT versions. This outcome is unsurprising given the heterogenous nature of the bilingual experience; an analysis at the population level may subsume individual response strategies, leaving unobservable, or latent subgroups of participants who exhibit common trajectories in their $D$ scores across test versions hidden within the larger population. Latent profile analysis (LPA) may therefore yield information about this hidden population structure to reveal how each IAT test version influenced a particular participant subgroup. The raw $D$ scores and the percent change in $D$ score between IAT test versions were taken as the manifest indicators to discriminate latent profiles.

LPA was conducted with the mclust statistical package (Scrucca et al., 2016) in R. To determine the best fit latent profile model, seven fit statistics were evaluated: log likelihood (LL), Akaike information criterion (AIC), Bayesian information criterion (BIC), sample-size-adjusted BIC (SSA-BIC), Lo-Mendell-Rubin likelihood ratio test (LMR), bootstrap likelihood ratio test (BLRT), and entropy, until the model with the lowest LL, AIC, BIC, and SSA-BIC values and significant LMR and BLRT values was identified (as per Nylund, Asparouhov, \& Muthén, 2007). The best fit model emerged when manifest indicators included $D$ scores in the bilingual, prescriptivist, and descriptivist versions and the percent change between them, indicating that the Spanish and English version $D$ scores were insufficiently informative for identifying latent subgroups. 
Figure 1. Profiles of Mexican-American Implicit Bias

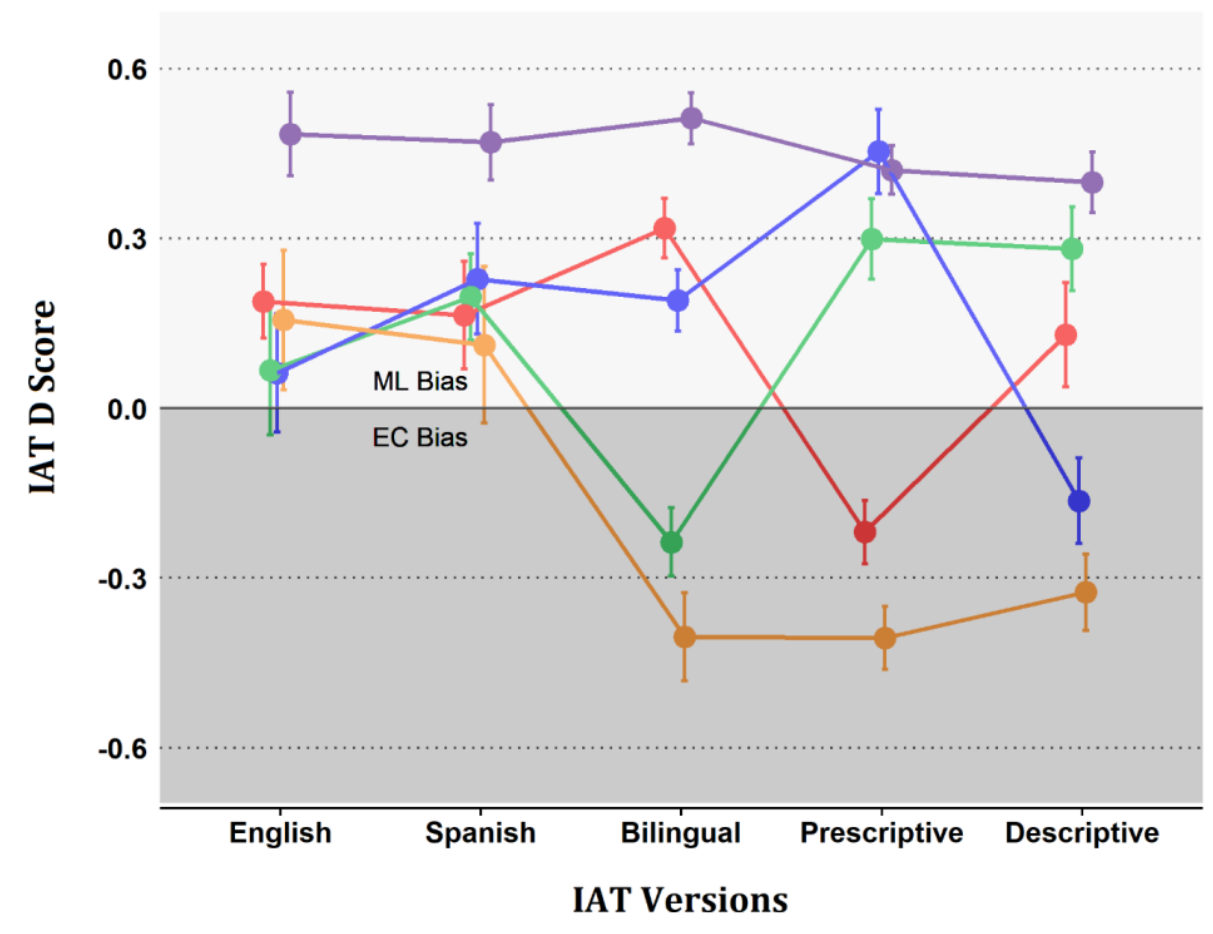

To reframe the relevance of LPA for our scenario in terms of more traditional analyses, we adopted the technique of validating the LPA classification in a second analysis of variance (Hart, Logan, Thompson, Kovas, McLoughlin, \& Petril, 2016). Participants were assigned to their most probably latent profile. Minimal error was assumed in this assignment as the best piecewise linear fit between models in the mclust entropy plot indicated an abrupt breakpoint at five profiles. The addition of latent profile as a factor with the factor of IAT test version now renders both factors and their interaction marginally to highly significant in an analysis of variance between subjects: IAT version $(\mathrm{F}(4,375)=2.30, p=0.058)$, latent profile $(\mathrm{F}(4,375)=35.83, p<0.001)$, and interaction $(\mathrm{F}(16,375)=7.13, p<0.001)$. Multiple regression analysis was used to test if the factors significantly predicted participants' $D$ scores. The two predictors explained $37.81 \%$ of the variance $(\mathrm{R} 2=.38, \mathrm{~F}(24,375)=11.11, p<0.001)$. The profile with consistently high ML bias was significantly different from all other profile groups (red $p=0.0030$; blue $p<0.001$; green $p<0.001$; yellow $p=0.014)$. In the interaction between latent profile and IAT version, all comparisons between the profiles with ML bias and EC bias were significantly different in the bilingual and prescriptive condition, whereas this distinction is lost in the descriptive condition. 


\subsection{Correlation Analysis}

Insight into how the bilinguals in each latent profile differ from one another can be gained by correlating participant background characteristics recorded in the initial surveys and pretests with their IAT $D$ scores. A Pearson's correlation was conducted to identify relationships between these factors (see Table 1). When ML bias was recorded for a particular profile in any of the IAT versions (see Figure 1), a significant positive association ("+") between $D$ score and the background characteristic implies that the trait may facilitate bias towards participants' own ethnicity for that profile; this orientation is the natural and anticipated response. When EC bias was displayed in any IAT version for a particular profile, a significant positive association reveals traits that contribute to a sense of 'false consciousness' (Jost \& Banaji, 1994); that is, a counterintuitive bias against participants' own ethnicity. The same logic applies to interpretation of significant negative associations (“-”): these traits facilitate ingroup devaluation when ML bias is reported for a particular subgroup and promote positive associations towards one's own ethnicity when EC bias is reported. On this basis, we attempt a preliminary sketch of profile members. The correlation that supports each supposition is referenced.

Participants who maintained consistently high ML bias (purple profile) may draw upon their strong network of Mexican Latino friends, colleagues, and authority figures (+ English \& Spanish IAT), perhaps to the exclusion of European Caucasians in their wider circle of friends (- English IAT). These participants hold warm feelings towards Spanish and a perception of proficiency in the language (+ bilingual IAT), yet risk overestimating their formal Spanish proficiency (- descriptive IAT).

On the other extreme, participants who shifted to EC bias in all bilingual conditions (orange profile) also count Mexican Latinos among their closest friends (+ English IAT) and codeswitch frequently with family and friends, as well as authority figures (+ Spanish IAT). However, they lack the same warm feelings towards Spanish and codeswitching (+ descriptive IAT). They do feel warm towards their own ethnic group (- bilingual IAT), except for when codeswitching takes place contrary to social norms (+ prescriptive IAT). At the same time, they negatively assess monolingual English stimuli items (+ prescriptive IAT). These results, as well as a predominance of European Caucasians among their peers in formal environments (- descriptive IAT), may explain why profile members appear particularly sensitive to pejorative monolingual views of bilingual language use. 


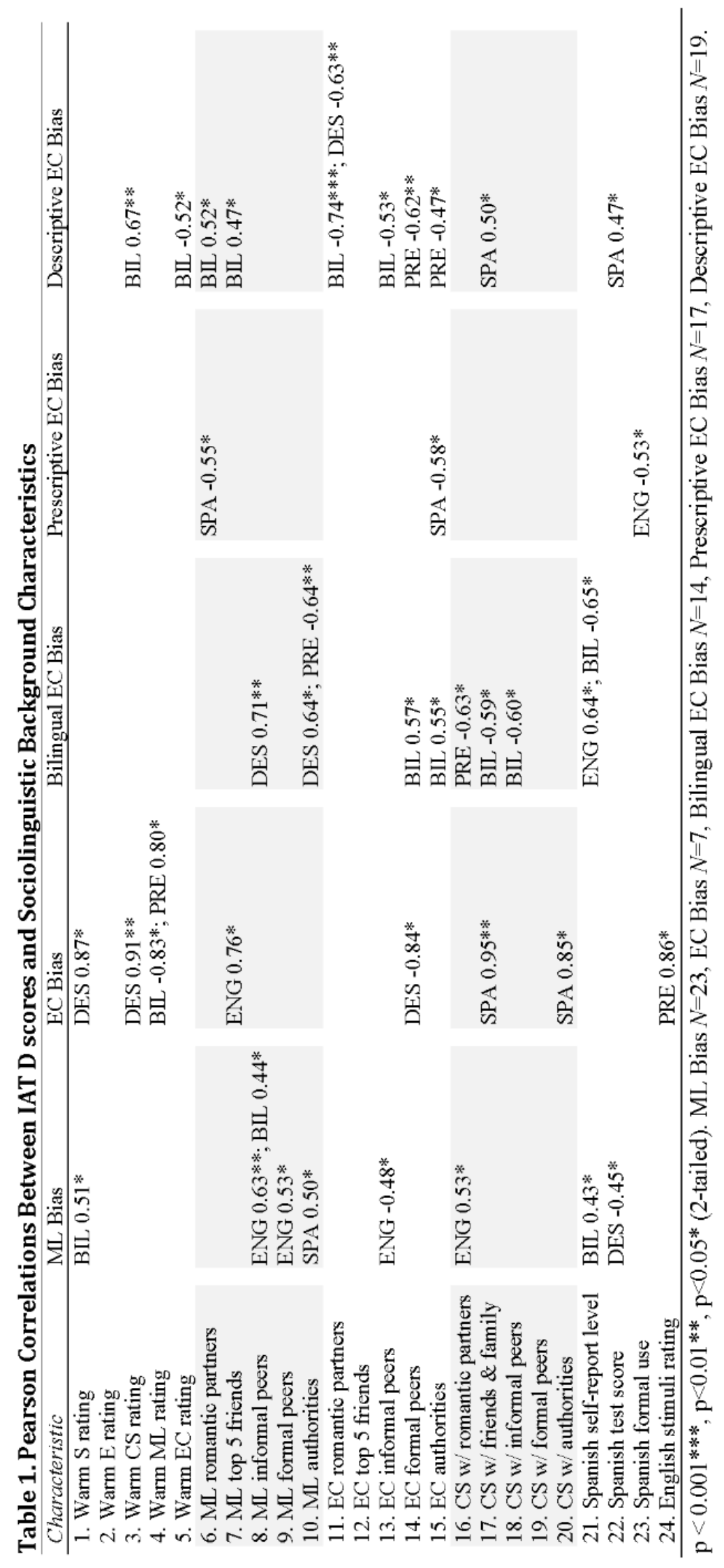


Participants who shifted to EC bias only in the bilingual condition (green profile) likewise codeswitch frequently with friends, family, and informal peer groups (bilingual IAT), but not with their romantic partners (- prescriptive IAT). Nonetheless, they perceive themselves to lack proficiency in Spanish (+ English IAT; - bilingual IAT). While possessing many Mexican Latino informal peers (+ descriptive IAT), these participants have few European Caucasian authority figures or peers in formal environments (+ bilingual IAT) and the Mexican Latino authorities they know may facilitate a prescriptivist mindset (- prescriptive IAT; + descriptive IAT). It could be this profile represents those bilinguals who codeswitch out of necessity rather than choice and acutely feel their lack of proficiency in Spanish, while maintaining positive views more generally of codeswitching.

It is not surprising that the least number of significant correlations are found among the profile that exhibited EC bias only in the prescriptive condition (red profile); this pattern of responses would seem natural in that one's own ethnicity is viewed negatively only when observed behavior contradicts expressed social norm. Unlike other profiles, all the correlations for these profile members are negative and limited to the English and Spanish IAT versions. The participants may encounter few European Caucasian authority figures (- Spanish IAT), yet use Spanish infrequently in formal settings (- English IAT) and have few Mexican Latino romantic partners (- Spanish IAT). These characteristics suggest profile members may experience few everyday conflicts between sociolinguistic expectations, which could account for their less idiosyncratic response to language attitude cues.

The final group of participants exhibit EC bias only in the descriptive condition (blue profile). They codeswitch frequently with family and friends (+ Spanish IAT) and hold warm feelings towards codeswitching (+ bilingual IAT), while still possessing high formal Spanish proficiency (+ Spanish IAT). Mexican Latinos comprise their closest group of friends and romantic partners (+ bilingual IAT). Profile members do not maintain warm feelings towards European Caucasians (- bilingual IAT) and may have little contact with authorities or peers of the ethnicity in both formal and informal environments (- prescriptive IAT; - bilingual IAT). This profile resembles bilinguals who may be less fully integrated into their multicultural surroundings; pejorative views of descriptivism were commonly expressed by survey respondents with recent ties to Mexico. Conflicting results are found for the prevalence of romantic relationships with European Caucasians, although this characteristic is specifically correlated with the shift to EC bias (- bilingual IAT; - descriptive IAT). 


\section{Discussion}

We predicted that the implicit associations expressed by Mexican-American bilinguals would shift between two linguistically distinct ethnic groups (Mexican Latinos and European Caucasians) in response to the social context in which an ethnically marked language practice — codeswitching — occurred. We observed this to be true, as prefacing the bilingual IAT version with a statement criticizing or condoning codeswitching resulted in a shift in the orientation of participants' implicit bias. Shifts in association strength were also observed when the controversial behavior was not preceded by any language attitude (the bilingual condition), suggesting that the social norms surrounding language use remain salient to participants even when not overtly primed.

In this manner, we have addressed considerations raised by previous studies (Danziger \& Ward, 2010; Ogunnaike et al., 2010): insofar as it can be interpreted from observing implicit associations, interacting in a language does not by proxy make an individual identify as a member of a linguistic ingroup for the duration of the task, and effects appear driven by the general indexical properties of language, rather than use of a specific language per se. Our participants share a sociocultural upbringing in which they were likely exposed to a similar set of distributional linguistic information. Therefore, the variation exhibited among profiles indicates that the IAT indeed reflects individual attitudes emerging from deep-rooted affective and conceptual processing, rather than the shallow linguistic processing of phrase-level statistical regularities (cf. Lynott, Kansal, Connell, \& O'Brien, 2012). In other words, it is the perception of the social appropriateness of a language variety that influences participant identification with the ethnicity associated with the language variety. The motivation for these effects suggested by Briley, Morris, \& Simonson (2005), that language selectively primes associations to promote effective social interaction, would appear well-founded.

It is important to note that substantial variation was observed throughout the population of subjects in the direction and extent to which association strengths shifted. Some Mexican-Americans endorsed the controversial language practice expressly when it was performed in violation of the prescriptive social norm. In considering the generalizability of our findings, it remains to be seen whether the same latent profiles would be upheld in a larger dataset or among participants from geographically or socially distinct regions. Nonetheless, the correlation of implicit and explicit measures can begin to shed light on the question of why implicit attitudes may be stable constructs for some respondents and state dependent for others. 


\section{References}

Anderson, J. A., Mak, L., Chahi, A. K., \& Bialystok, E. (2018). The language and social background questionnaire: Assessing degree of bilingualism in a diverse population. Behavior research methods, 50(1), 250-263.

Banaji, M. R. (2013). The Implicit Association Test at age 7: A methodological and conceptual review. Social psychology and the unconscious: The automaticity of higher mental processes, 265.

Boztepe, E. (2005). Issues in code-switching: competing theories and models. Teachers College. Columbia University Working Papers in TESOL \& Applied Linguistics, 3 (2).

Briley, D. A., Morris, M. W., \& Simonson, I. (2005). Cultural chameleons: Biculturals, conformity motives, and decision making. Journal of Consumer Psychology, 15(4), 351-362.

Danziger, S., \& Ward, R. (2010). Language changes implicit associations between ethnic groups and evaluation in bilinguals. Psychological Science, 21(6), 799.

De Fina, A. (2007). Codeswitching and the construction of ethnic identity in a community of practice. Language in society, 36(3), 371-392.

Drake, G. F. (1977). The role of prescriptivism in American linguistics, 1820-1970 (Vol. 13). John Benjamins Publishing.

Genesee, F. (1984). The social-psychological significance of bilingual code switching for children. Applied Psycholinguistics, 5(1), 3-20.

Greenwald, A. G., Nosek, B. A., \& Banaji, M. R. (2003). Understanding and using the implicit association test: I. An improved scoring algorithm. Journal of personality and social psychology, 85(2), 197.

Hart, S. A., Logan, J. A., Thompson, L., Kovas, Y., McLoughlin, G., \& Petrill, S. A. (2016). A latent profile analysis of math achievement, numerosity, and math anxiety in twins. Journal of educational psychology, 108(2), 181.

Jost, J. T., \& Banaji, M. R. (1994). The role of stereotyping in system-justification and the production of false consciousness. British journal of social psychology, 33(1), 127.

Judd, C. M., Park, B., Ryan, C. S., Brauer, M., \& Kraus, S. (1995). Stereotypes and ethnocentrism: Diverging interethnic perceptions of African American and White American youth. Journal of personality and social psychology, 69(3), 460.

Kharkhurin, A. V., \& Wei, L. (2015). The role of code-switching in bilingual creativity. International Journal of Bilingual Education and Bilingualism, 18(2), 153-169.

Lynott, D., Kansal, H., Connell, L., \& O'Brien, K. (2012). Modelling the IAT: implicit association test reflects shallow linguistic environment and not deep personal attitudes. In Proceedings of the Annual Meeting of the Cognitive Science Society (Vol. 34, No. 34).

MacSwan, J. (2000). The architecture of the bilingual language faculty: Evidence from intrasentential code switching. Bilingualism: language and cognition, 3(1), 37-54. 
Masyn, K. E. (2013). 25 latent class analysis and finite mixture modeling. In The Oxford handbook of quantitative methods (p. 551). Oxford University Press, Oxford.

McLarnon, M. J., \& O’Neill, T. A. (2018). Extensions of auxiliary variable approaches for the investigation of mediation, moderation, and conditional effects in mixture models. Organizational Research Methods, 21(4), 955-982.

Molinsky, A. (2007). Cross-cultural code-switching: The psychological challenges of adapting behavior in foreign cultural interactions. Academy of Management Review, $32(2), 622-640$.

Myers-Scotton, C. (1995). Social motivations for codeswitching: Evidence from Africa. Oxford University Press.

Nosek, B. A., Greenwald, A. G., \& Banaji, M. R. (2005). Understanding and using the Implicit Association Test: II. Method variables and construct validity. Personality and Social Psychology Bulletin, 31(2), 166-180.

Nylund, K. L., Asparouhov, T., \& Muthén, B. O. (2007). Deciding on the number of classes in latent class analysis and growth mixture modeling: A Monte Carlo simulation study. Structural equation modeling: A multidisciplinary Journal, 14(4), 535-569.

Ogunnaike, O., Dunham, Y., \& Banaji, M. R. (2010). The language of implicit preferences. Journal of Experimental Social Psychology, 46(6), 999-1003.

Oswald, F. L., Mitchell, G., Blanton, H., Jaccard, J., \& Tetlock, P. E. (2015). Using the IAT to predict ethnic and racial discrimination: Small effect sizes of unknown societal significance.

Park, J., \& Yu, H. T. (2018). Recommendations on the sample sizes for multilevel latent class models. Educational and Psychological Measurement, 78(5), 737-761.

Pettigrew, T. F. (1998). Intergroup contact theory. Annual review of psychology, 49(1), 65-85.

Scrucca, L., Fop, M., Murphy, T. B., \& Raftery, A. E. (2016). mclust 5: clustering, classification and density estimation using Gaussian finite mixture models. The $R$ journal, 8(1), 289. 